\title{
MEMBRANE-RECEPTOR COMPLEX DYSFUNCTION AS A NEW TARGET FOR THE NEUROTROPHIC THERAPY OF ISCHEMIC STROKE
}

\author{
Volodymyr Lychko ${ }^{1}$ \\ ${ }^{1}$ 1Department of Neurosurgery and Neurology, Medical Institute of Sumy State University, Sumy, Ukraine \\ volodlychko@gmail.com \\ ORCID: http://orcid.org/0000-0001-5518-5274
}

ARTICLE INFO

ABSTRACT

Article history:

Received date 29.09.2020

Accepted date 21.10.2020

Published date 30.10 .2020

The aim of the study was the $\beta$-adrenergic activity of peripheral blood erythrocyte mem-

Section:

Practical medicine

DOI

$10.21303 / 2313-8416.2020 .001450$ branes in the acute period of ischemic stroke (IS).

Peripheral blood erythrocytes are the most informative biological tissue for studying many pathogenetic mechanisms, and the work of their membrane-receptor complex (MRC) can very well reflect the functional state of the whole organism, one of the most important criteria for assessing is the adrenergic activity of cytoplasmic membranes in the pathogenesis of IS.

This study solves the problem of the finding of the features of changes in the adrenocepKEYWORDS tors system in the acute period of IS in the dynamics of the treatment of humans cryopreserved cord blood serum (CCBS).

\section{resistance}

membrane

$\beta$-adrenergic receptor

ischemic stroke

The main scientific results: the obtained results indicate a reduced ability of adrenocepdysfunction tors (ARs) on erythrocyte membranes to bind blockers due to the desensitisation effect, which is observed from the 1st day of the disease.

In the onset of IS, there is a significant increase in the values of $\beta$-adrenergic activity of membranes ( $\beta$-ARM) of erythrocytes in 2.4 times compared with the control. Maximum levels of the indicator $(42.43 \pm 3.64 \mathrm{CU})$ are observed in patients with initially severe disease, which indicates significant stress of the sympathoadrenal system (SAS) in these patients.

On the 10th day of the disease, there was a decrease in $\beta$-ARM relative to the values obtained during hospitalisation. Still, comparing the value of the indicator on the 10th day of IS in both groups, there was an apparent, more significant decrease in $\beta$-ARM in the 2nd group of patients, which additionally received CCBS.

The use of CCBS contributes to a more pronounced degree of recovery in neurological functions $(6.9 \%)$ and faster stabilisation of the structure of MRCs in erythrocytes in the form of reducing the values of $\beta$-ARM to $16.61 \pm 2.86 \mathrm{CU}(\mathrm{p}<0.05)$.

The area of practical use of the research results: the results of the study can be used to diagnose and treat of the IS.

An innovative technological product: A comprehensive approach to assess the $\beta$-ARM of peripheral blood erythrocytes in patients with IS by determining the degree of changes in osmotic resistance of erythrocytes (ORE) under the action of adrenergic drugs (AD) is informative and can be used to diagnose of IS. The use of neurotrophic factors is a potentially new method of treating this pathology.

Scope of application of the innovative technological product: in the clinical practice of neurological departments for the treatment of patients with strokes.

(C) The Author(s) 2020. This is an open access article under the CC BY license http://creativecommons.org/licenses/by/4.0).

\section{Introduction}

In recent years, the pathogenetic concept of ischemic stroke (IS) has been formulated in the form of rapid specific structural and functional changes in brain tissue [1, 2]. An extremely important and influential factor that directly affects the severity of the disease and determines its outcome is the functional state of the membrane-receptor complex (MRC) of cells not only in the affected area but also throughout the body [3, 4].

The cascade of pathological biochemical reactions in the focus of hypoxia, namely oxidative stress, glutamate excitotoxicity, local inflammation and others, primarily leads to damage to the 
outer cytoplasmic membranes of cells and their receptor apparatus with the rapid development of membrane-receptor dysfunction [5-7].

Structural and functional changes in many parameters of brain tissue are correlated mainly with violations of the electrophysical characteristics of cells in the acute period of IS, which is quite informatively visualised using spectrophotometric methods [8]. Under the conditions of deployment of almost any type of pathology, the presence of stable changes in the hydrated environment of biological molecules of damaged tissues, which radically change cell metabolism [9].

During IS, hyper-activation of numerous sympathetic centres occurs, and the closely related sympathoadrenal system (SAS) functions through $\beta$-adrenoceptors ( $\beta$-ARc) [10].

\section{1. The object of research}

The object of the study was the $\beta$-adrenergic reactivity of peripheral blood erythrocyte membranes $(\beta$-ARM) in the acute period of IS.

\section{2. Problem description}

Today, peripheral blood erythrocytes are the most informative biological tissue for studying pathogenetic mechanisms, and their MRC can very well reflect the functional state of the whole organism. One of the most important criteria for assessing, which is the adrenergic activity of cytoplasmic membranes, also plays a leading role in pathogenesis [11, 12].

In the treatment tactics of the acute period of the disease, not enough attention is paid to the methods of drug correction of membrane-receptor dysfunction, which could clearly have a positive effect on the outcome of the disease.

\section{3. The proposed solution to the problem}

Of particular interest in this study is the investgation of the effect of targeted, namely membrane-protective therapy on the above mechanisms of pathogenesis of IS.

The use of neurotrophic factors is a potentially new method of treating some cardiovascular diseases and their complications, which is IS [13-15].

One such drug is «Cryocord», which is a human cryopreserved cord blood serum (CCBS), developed by the Institute of Cryobiology and Cryomedicine of the National Academy of Sciences of Ukraine and approved by the Ministry of Health for clinical use.

The aim of the work was a comprehensive study of the features of changes in the adrenoceptors system in the acute period of IS in the dynamics of the treatment of human CCBS.

\section{Materials and methods of the research}

Changes in the osmotic resistance of erythrocytes (ORE) under the action of $\beta$-blockers $(\beta-\mathrm{AB})$ were determined by photoelectron colourimetry, which is based on the fact of inhibition of hemolysis placed in a hypo-osmotic medium in the presence of $\beta$-AB propranolol at a wavelength of $540 \mathrm{~nm}[16]$.

In a test sample with a similar solution of hypoosmotic buffer was added $\beta$ - $A B$, which, by binding to $\beta$-ARc membranes, reduced the degree of hemolysis of erythrocytes. Increased ORE under the influence of $\beta-A B$ is associated with the regulation of $\mathrm{Na}^{+} / \mathrm{H}^{+}$- and $\mathrm{Na}^{+} / \mathrm{K}^{+}$-exchanges of the cell.

The extinction value of the test sample was expressed as a percentage of the extinction value of the control sample, and the percentage units were taken as $\mathrm{CU} \beta$-workstation:

$$
\beta-\mathrm{ARM}=\left(E_{01}+E_{02} / E_{k 1}+E_{k 2}\right) \times 100 \%,
$$

where $\beta$-ARM - the value of $\beta$-ARM, CU; $E_{01}$ and $E_{02}$ - values of the optical density of the studied samples; $\mathrm{E}_{\mathrm{k} 1}$ and $\mathrm{E}_{\mathrm{k} 2}$ - values of the optical density of control samples.

Average values of $\beta$-ARM in $93 \%$ of almost healthy individuals are in the range from 2 to $20 \mathrm{CU}$, which reflects the increase in ORE as a result of binding of $\beta$-AB to functioning $\beta$-ARs membranes. The decrease in $\beta$-ARM is explained by a reduction in the number of sensitive to adrenergic drugs (AD) $\beta$-ARs due to their desensitisation. At the same time, binding of the blocker decreases, the osmotic resistance also decreases that is reflected in the value of $\beta$-APM, which exceeds $20 \mathrm{CU}$. The ORE values were recorded using a photoelectron colourimeter. 
Pharmacological studies were performed in vivo. Statistical processing of the results of experimental studies was carried out using the program Statistics 6.0.

The work was based on the materials of a comprehensive examination of 350 patients with the first in the life of IS on the 1st, 10th and 21st day of the disease. There were 185 males (52.8\%) and 165 females $(47.2 \%)$. The mean age of the examined patients was $65.2 \pm 0.7$ years (range from 46 to 79 years). As a comparison group, an additional 30 relatively healthy individuals were examined (17 men and 13 women, mean age $-60.4 \pm 0.8$ years), who are homogeneous in sex and age with a group of patients with IS.

Depending on the prescribed drug therapy, all patients were randomly divided into two groups before treatment: the first $(n=175)$ - undifferentiated therapy + differentiated with the appointment of acetylsalicylic acid (ASA); the second $(n=175)$ - undifferentiated therapy + differentiated with the appointment of ASA, which was supplemented by the introduction of $1 \mathrm{ml}$ of intravenous CCBS solution for 10 days.

The severity of the condition and the degree of neurological deficit were objectified using the stroke scale of the US National Institutes of Health (NIHSS) with a score of 15 indicators, expressed in points in the first hours of the disease, in the dynamics of treatment on the 10th and 21 st day.

Based on this, all patients were divided into two clinical subgroups: A $(n=183)$ - patients in a state of moderate severity (mean score on the NIHSS scale in the 1st group 11.74 \pm 0.33 , in the $2 \mathrm{nd}-11.61 \pm 0.22)$; $\mathrm{B}(\mathrm{n}=167)$ - patients with severe IS (mean score on the NIHSS scale in the 1 st group $-23.11 \pm 0.37$, in the 2 nd $-24.06 \pm 0.29$ ).

\section{Results}

The total value of $\beta$-ARM in the control group was $15.3 \pm 4.4 \mathrm{CU}$, which corresponds to the literature data [16]. The values of the indicator in the group of all patients with IS on the 1st, 10th and 21st day of the disease were significantly higher than the control (Table 1).

Table 1

General values of $\beta$-ARM in patients with IS in the dynamics of treatment, $\mathrm{CU}$

\begin{tabular}{cccc}
\hline \multirow{2}{*}{ Group of patients } & & $\boldsymbol{\beta}$-ARM (CU) & 21st day \\
\cline { 2 - 4 } & 1st day & 10th day & $19.73 \pm 3.48$ \\
\hline All & $36.3 \pm 3.72$ & $25.68 \pm 3.32$ & $23.31 \pm 3.18$ \\
1 group & $35.88 \pm 2.94$ & $28.67 \pm 3.52$ & $16.61 \pm 2.86^{*}$ \\
2 group & $36.62 \pm 3.24$ & $23.22 \pm 2.63$ & $15.34 .4 \pm$
\end{tabular}

Note: all indicators are reliable $(p<0.05)$ relative to the control group; $*-p<0.05$ before and after treatment within the group

The results indicate a reduced ability of ARs on erythrocyte membranes to bind blockers due to the desensitisation effect, which is observed from the 1st day of the disease and is consistent with the literature on MRC damage in response to hypoxia [11].

On the 10th day of the disease, there was a decrease in $\beta$-ARM relative to the values obtained during hospitalisation. Still, comparing the value of the indicator on the 10th day of IS in both groups, there was an apparent more significant decrease in $\beta$-ARM in the 2nd group of patients, which additionally received CCBS.

A similar tendency to decrease the values of $\beta$-ARM was also observed in patients on the 21 st day of the disease. The apparent difference in costs between the groups indicates a more positive dynamics of the restoration of the functioning of MRC cells under conditions of hypoxia in patients who additionally received CCBS.

The dynamics of changes in $\beta$-ARM depending on the severity of IS is presented in Fig. 1.

Thus, the indicators of $\beta$-ARMs of the control group were within normal limits (the average group value was $15.3 \pm 4.4 \mathrm{CU}$ ), which according to the method [17] corresponds to the normal $\beta$-ARMs (2-20 CU). In patients of subgroup A with moderate IS severity, the index exceeded 
normal values by 1.97 times, which is characteristic of the average degree of $\beta$-ARM $(21-40 \mathrm{CU})$ and indicates a reduced ability of ARs erythrocyte membranes to bind a blocker due to the desensitisation effect. A similar situation was observed with erythrocytes of patients of subgroup B with severe disease, where the rate of $\beta$-ARM exceeded average values by 2.8 times and was characterised by a low degree of $\beta$-ARM ( $>41 \mathrm{CU})$.

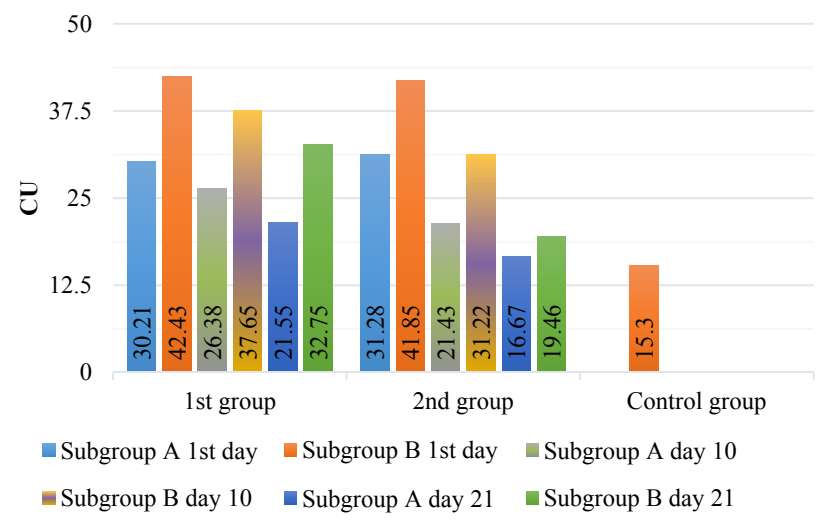

Fig. 1. Dynamics of $\beta$-ARM values in patients with IS depending on the severity of the disease (CU)

In patients of the 2nd group with severe IS, lower values of $\beta$-ARM on the 10 th day were also found in comparison with the corresponding indicators of the 1st group. In percentage terms, the values of $\beta$-ARM in patients with severe IS on the 10th day decreased in the 2nd group by $25.4 \%$, while in the 1 st - only by $11.3 \%$.

If we evaluate the dynamics of the value of $\beta$-ARM on the 21 st day of treatment, we can trace positive changes in the indicator, which approached the control values, in almost all groups of patients. This was especially evident in patients of the 2 nd group in moderate condition, who additionally received CCBS. The maximum values of this indicator on the 21st day were found in patients with severe IS $(32.75 \pm 2.72 \mathrm{CU})$, who received standard treatment, which is still more than two times higher than the control values.

\section{Discussion of research results}

This method has already been used to confirm the degree of desensitization of ARs cell membranes during such pathological conditions as heart failure, arterial hypertension, atherosclerosis and others [11]. These results demonstrate that substantial changes in the functioning of erythrocyte MRC can determine the outcome of the disease. Similar data were also obtained in our study with patients in the acute period of IS. This is evidenced by statistical processing.

Thus, the correlation analysis (Fig. 2) of the relationship between $\beta$-ARM values and the total clinical score on the NIHSS scale showed their direct relationship $(r=+0.93 ; p<0.05)$. In the dynamics of observation between ones, there was a correlation, which did not lose its strength and direction $(\mathrm{r}=+0.81 ; \mathrm{p}<0.05)$.

There was a significant inverse correlation between the levels of $\beta$-ARM and the age of patients with IS $(r=-0.63 ; p<0.05)$, which confirms the age-dependent mechanisms of SAS depletion.

The reduced ability of ARs on erythrocyte membranes to bind blockers due to the desensitisation effect due to MRC damage is evidenced by the established direct, reliable relationship between the area of the infarct zone and the values of $\beta$-ARM $(r=+0.88 ; p<0.05)$.

In the course of the study, causal relationships were established between changes in the structural and functional characteristics of brain tissue, indicators of the functional state of SAS, the severity of patients and the volume of the affected area, indicating their pathogenetic significance in the development and course of IS.

Thus, the rate of $\beta$-ARM on the 1st day of the disease can reach $36.62 \pm 3.24 \mathrm{CU}$ in severe patients. Further, an increase from $41.85 \pm 2.98$ to $42.43 \pm 3.64 \mathrm{CU}$ in the dynamics of IS should be considered a sign of a critical degree of dysfunction of the adrenergic receptor system. 


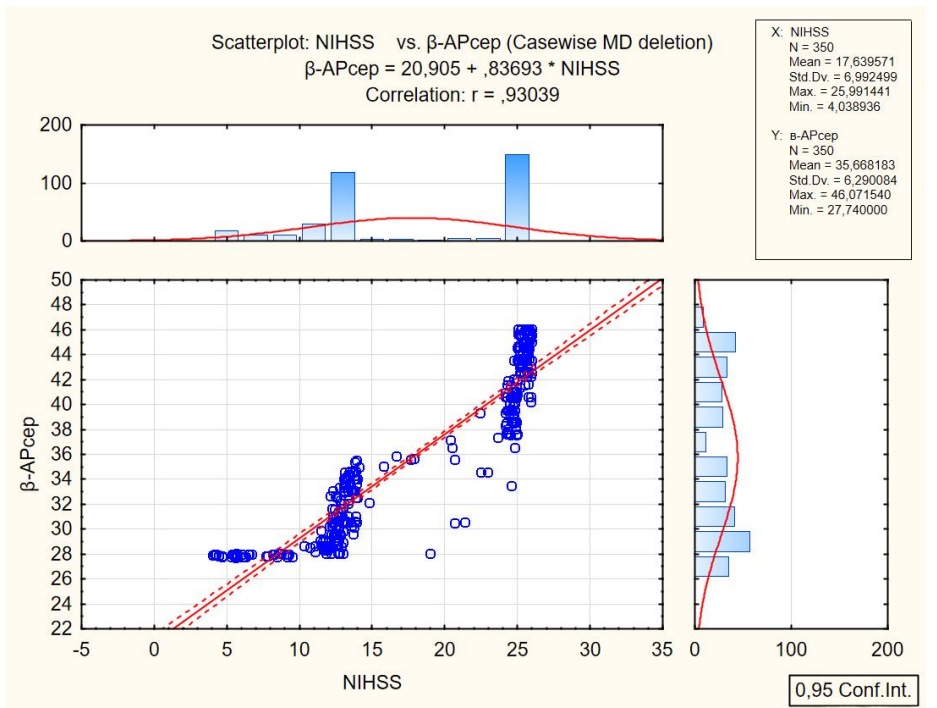

Fig. 2. Correlation between $\beta$-ARM values and the degree of the neurological deficit on the NIHSS scale in patients with IS during hospitalisation.

The results of the study showed that the developed comprehensive approach for the assessment of $\beta$-ARM of peripheral blood erythrocytes in patients with IS by determining the degree of changes in ORE under the action of AD is informative. The application of this approach showed that the deviations of indicators that were induced by adrenergic substances in vitro, are a manifestation of the specific response of cells to the action of biologically active substances and depend on the functional state of the SAS.

In patients with such concomitant pathology as acute heart failure, liver dysfunction with an increase in the level of hepatic transaminases by more than 3 times, renal failure with a creatinine content in the blood (over $132.6 \mu \mathrm{mol} / \mathrm{l}$ ), oncological diseases during the past five years, convulsive syndrome, blood and acute infectious diseases are not recommended for the investigation of $\beta$-ARM due to significant distortion of the results.

\section{Conclusions}

1. On the onset of IS, there is a significant increase in the values of $\beta$-ARM in 2.4 times compared with the control. Maximum levels of the indicator (42.433.64 $\pm \mathrm{CU}$ ) are observed in patients with initially severe disease (neurological deficit on the NIHSS scale of 21 points and above), which indicates significant stress of SAS in these patients.

2. The revealed direct correlation between the total clinical score and the level of $\beta$-ARM in patients in the acute period of IS $(r=+0.93$; $p<0.05)$ without changing the direction in the dynamics of the disease indicates a stronger lesion of MRC in severe patients.

3. Additional inclusion in the complex therapy of patients with IS by CCBS contributes to a more pronounced degree of recovery of neurological functions $(6.9 \%)$ and faster stabilisation of the structure of MRC of erythrocytes in the form of reducing $\beta$-ARM to $16.61 \pm$ $\pm 2.86 \mathrm{CU}(\mathrm{p}<0.05)$.

\section{Conflict of interest}

The authors declare that they have no conflicts of interest.

\section{References}

[1] De Oliveira, M. R., Duarte, A. R., Chenet, A. L., de Almeida, F. J. S., Andrade, C. M. B. (2019). Carnosic Acid Pretreatment Attenuates Mitochondrial Dysfunction in SH-SY5Y Cells in an Experimental Model of Glutamate-Induced Excitotoxicity. Neurotoxicity Research, 36 (3), 551-562. doi: http://doi.org/10.1007/s12640-019-00044-8

[2] Zhang, Z., Yan, J., Shi, H. (2016). Role of Hypoxia Inducible Factor 1 in Hyperglycemia-Exacerbated Blood-Brain Barrier Disruption in Ischemic Stroke. Neurobiology of Disease, 95, 82-92. doi: http://oi.org/10.1016/j.nbd.2016.07.012

[3] Bivard, A., Parsons, M. (2018). Tissue is more important than time: insights into acute ischemic stroke from modern brain imaging. Current Opinion in Neurology, 31 (1), 23-27. doi: http://doi.org/10.1097/wco.0000000000000520 
[4] Lees, K. R., Bornstein, N., Diener, H.-C., Gorelick, P. B., Rosenberg, G., Shuaib, A. (2013). Results of Membrane-activated Chelator Stroke Intervention Randomized Trial of DP-b99 in Acute Ischemic Stroke. Stroke, 44 (3), 580-584. doi: http:// doi.org/10.1161/strokeaha.111.000013

[5] Boehme, A. K., McClure, L. A., Zhang, Y., Luna, J. M., Del Brutto, O. H., Benavente, O. R., Elkind, M. S. V. (2016). Inflammatory Markers and Outcomes After Lacunar. Stroke Levels of Inflammatory Markers in Treatment of Stroke Study. Stroke, 47 (3), 659-667. doi: http://doi.org/10.1161/strokeaha.115.012166

[6] Khoshnam, S. E., Winlow, W., Farzaneh, M., Farbood, Y., Moghaddam, H. F. (2017). Pathogenic mechanisms following ischemic stroke. Neurological Sciences, 38 (7), 1167-1186. doi: http://doi.org/10.1007/s10072-017-2938-1

[7] Zis, O., Zhang, S., Dorovini-Zis, K., Wang, L., Song, W. (2014). Hypoxia Signaling Regulates Macrophage Migration Inhibitory Factor (MIF) Expression in Stroke. Molecular Neurobiology, 51 (1), 155-167. doi: http://doi.org/10.1007/s12035014-8727-4

[8] Beneduci, A., Cosentino, K., Romeo, S., Massa, R., Chidichimo, G. (2014). Effect of millimetre waves on phosphatidylcholine membrane models: a non-thermal mechanism of interaction. Soft Matter, 10 (30), 5559-5567. doi: http://doi.org/10.1039/ c4sm00551a

[9] Gapeyev, A. B., Sokolov, P. A., Chemeris, N. K. (2001). Response of membrane-associated calcium signaling systems of the cell to extremely low-frequency external signals with different waveform parameters. Electro- and Magnetobiology, 20 (1), 107-122. doi: http://doi.org/10.1081/jbc-100103163

[10] Hama, S., Murakami, T., Yamashita, H., Onoda, K., Yamawaki, S., Kurisu, K. (2016). Neuroanatomic pathways associated with monoaminergic dysregulation after stroke. International Journal of Geriatric Psychiatry, 32 (6), 633-642. doi: http:// doi.org/10.1002/gps.4503

[11] Hausdorff, W. P., Caron, M. G., Lefkowitz, R. J. (1990). Turning off the signal: desensitization of $\beta$-adrenergic receptor function. The FASEB Journal, 4 (11), 2881-2889. doi: http://doi.org/10.1096/fasebj.4.11.2165947

[12] Johnson, J. A., Terra, S. G. (2002). Beta-adrenergic receptor polymorphisms: Cardiovascular disease associations and pharmacogenetics. Pharmaceutical Research, 19 (12), 1779-1787. doi:-http://doi.org/10.1023/a:1021477021102

[13] Foldvari, M., Chen, D. W. (2016). The intricacies of neurotrophic factor therapy for retinal ganglion cell rescue in glaucoma: a case for gene therapy. Neural Regeneration Research, 11 (6), 875-877. doi: http://doi.org/10.4103/1673-5374.184448

[14] Liu, X., Ye, R., Yan, T., Yu, S. P., Wei, L., Xu, G. et. al. (2014). Cell based therapies for ischemic stroke: From basic science to bedside. Progress in Neurobiology, 115, 92-115. doi: http://doi.org/10.1016/j.pneurobio.2013.11.007

[15] Patel, R. A. G., McMullen, P. W. (2017). Neuroprotection in the Treatment of Acute Ischemic Stroke. Progress in Cardiovascular Diseases, 59 (6), 542-548. doi: http://doi.org/10.1016/j.pcad.2017.04.005

[16] Tsirkin, V. I., Gromova, M. A., Kolchina, D. A. (2008). Otsenka adrenoreaktivnosti eritrotsitov, osnovannaya na sposobnosti povyishat skorost agglyutinatsii eritrotsitov. Fundamentalnyie issledovaniya, 7, 59-60.

[17] Malakhov, V. O., Nosatov, A. V., Fisun, A. I., Sirenko, S. P., Bilous, O. I. (2016). Pat. No. 108233 UA. Sposib kompleksnoho likuvannia porushen mozkovoho krovoobihu. MPK: G01N 33/49 (2006.01), A61B 5/145 (2006.01). No. u 201600029; declareted: 04.01.2016; published: 11.07.2016, Bul. No. 13. 